\title{
Minimum Bias and Underlying Event studies with a Monte Carlo tune for pp events with the ATLAS detector
}

\author{
Emily Nurse ${ }^{* \dagger}$ \\ University College London \\ E-mail: nurse@hep.ucl.ac.uk
}

Charged particle distributions from $p p$ collisions at $\sqrt{s}=0.9$ and $7 \mathrm{TeV}$ measured with the ATLAS detector are presented. The distributions are shown in diffraction suppressed and diffraction enhanced minimum bias event samples. The fraction of events in the diffraction enhanced sample is used to constrain the relative diffractive cross section. Underlying event distributions, where the charged particle density in a region transverse to the hard interaction is plotted as a function of the transverse momentum of the leading charged particle, are also presented. In addition a new ATLAS tune to the diffraction suppressed minimum bias and underlying event data is presented.

35th International Conference of High Energy Physics - ICHEP2010,

July 22-28, 2010

Paris France

*Speaker.

$\dagger$ on behalf of the ATLAS collaboration. 


\section{Introduction}

The dominant component of the inelastic cross section at the LHC comes from soft QCD processes that are often classified into single-diffractive dissociation (SD), where a Pomeron is exchanged between the protons and one of the protons dissociates, double-diffractive dissociation (DD), where both of the protons dissociate, and non-diffractive (ND) events. The signature of $\mathrm{SD}(\mathrm{DD})$ events is a rapidity gap on one side (the centre) of the detector. Studies of these processes are often known as "minimum bias" (MB) studies, indicating that some minimal criteria are made to select inelastic collisions.

The underlying event (UE) is a term used to describe everything that happens in a hadronhadron interaction, other than the primary parton-parton interaction. The UE includes additional (softer) parton-parton interactions and beam-beam remnants.

The description of these soft inclusive processes in Monte Carlo (MC) generators is typically done via phenomenological models, with parameters that must be tuned to the data. This paper presents measurements made with the ATLAS detector of MB and UE distributions for $\sqrt{s}=0.9$ and $7 \mathrm{TeV} p p$ collisions. In addition, a tune of the PYTHIA6 event generator [1] to this data is presented.

\section{Detector}

The ATLAS detector [2] components relevant to these measurements include the Inner Detector tracking system, which allows the tracks of charged particles to be reconstructed within the pseudorapidity range $|\eta|<2.5$, and the Minimum Bias Trigger Scintillator (MBTS) disks, which trigger on the presence of charged particles in the region $2.09<|\eta|<3.84$, on both sides of the detector. All analyses in this paper were triggered by the presence of activity on either of the MBTS disks.

\section{Results}

\subsection{Diffraction suppressed minimum bias}

In order to perform a tune of the ND parameters in PYTHIA6, with reduced systematic effects due to a poor modelling of the diffractive processes, a diffraction suppressed event sample is defined. Events in this diffraction suppressed sample are required to contain at least six charged particles with transverse momentum $p_{\mathrm{T}}>500 \mathrm{MeV}$ and $|\eta|<2.5$, and the distributions of all charged particles passing these kinematic cuts are studied. Since diffractive events usually contain a small number of central charged particles, this requirement significantly reduces the diffractive component of the sample. According to PYTHIA6(PYTHIA8) the remaining diffractive component is $0.4 \%(10 \%)$ at $\sqrt{s}=7 \mathrm{TeV}$.

Figure 1 shows the $n_{\mathrm{ch}}, p_{\mathrm{T}}$ and $\left\langle p_{\mathrm{T}}\right\rangle \mathrm{v} s . n_{\mathrm{ch}}$ distributions at $\sqrt{s}=7 \mathrm{TeV}$, where $n_{\mathrm{ch}}$ is the number of charged particles with $p_{\mathrm{T}}>500 \mathrm{MeV}$ and $|\eta|<2.5$. The first two distributions are normalised to the number of selected events. The data have been fully corrected for detector effects. Comparisons with $\sqrt{s}=0.9 \mathrm{TeV}$ data are not shown, the main features are similar but the agreement with $\mathrm{MC}$ is generally better. 


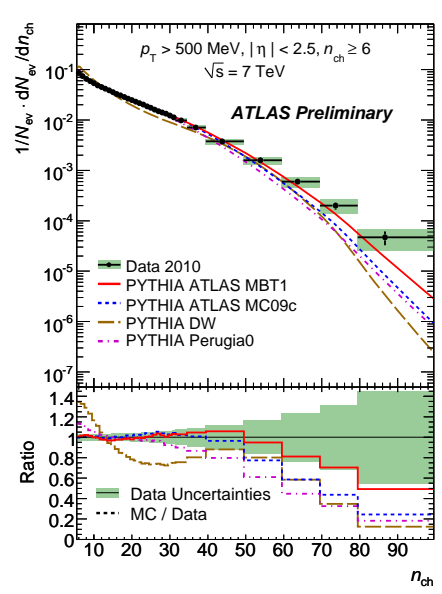

(a)

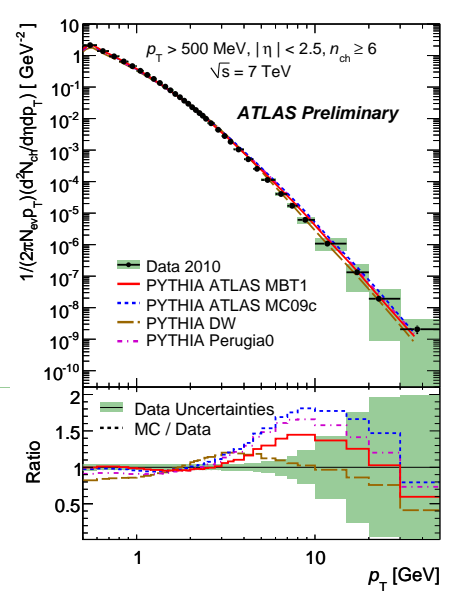

(b)

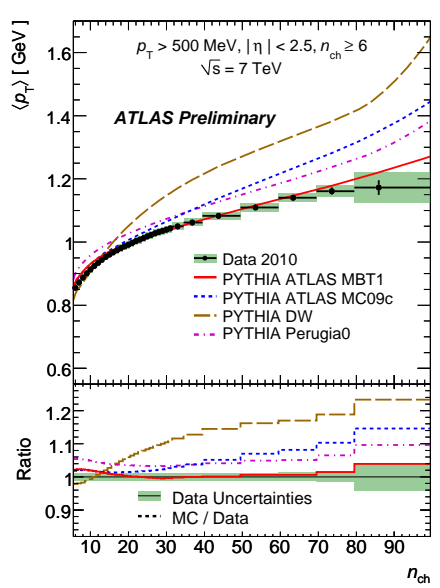

(c)

Figure 1: Multiplicity (a), $p_{\mathrm{T}}$ (b) and $\left\langle p_{\mathrm{T}}\right\rangle$ vs. $n_{\mathrm{ch}}$ (c) distributions for charged particles in a diffraction suppressed event sample.

The data are compared to various pre-LHC PYTHIA6 tunes, as well as the new ATLAS tune, AMBT1. All pre-LHC tunes under-predict the high $n_{\mathrm{ch}}$ tail, which is improved in AMBT1. This is mostly achieved by reducing the parameter that sets the width of the inner Gaussian in the proton matter distribution (PARP(84)). In PYTHIA6 the distribution is described by a double Gaussian. Reducing the width of this inner Gaussian means that head on collisions are more likely and therefore increases the amount of activity.

The medium-high $p_{\mathrm{T}}$ tail and the $\left\langle p_{\mathrm{T}}\right\rangle$ vs. $n_{\mathrm{ch}}$ distribution are over-estimated by all pre-LHC tunes. AMBT1 improves the discrepancy by increasing the parameter PARP(77), which is a colourreconnection suppression factor for high momentum hadrons (colour-reconnection leads to less hadrons for a given parton final state). A full description of this data analysis and all the parameters changed in the MC tune can be found in Ref. [3].

\subsection{Underlying Event}

The UE distributions aim to measure the activity of an event in a region transverse to the hard scatter. In this analysis the direction of the hard scatter is defined to be the direction of the charged particle with $|\eta|<2.5$ with the largest $p_{\mathrm{T}}$ in the event ( $p_{\mathrm{T}}^{\text {lead }}$ ). The detector is then split into three regions in $\phi$ : the toward region $\left(|\Delta \phi|<60^{\circ}\right)$, the away region $\left(|\Delta \phi|>120^{\circ}\right)$ and the transverse region $\left(60^{\circ}|\Delta \phi|<120^{\circ}\right)$, where $\Delta \phi$ is the distance in $\phi$ from leading charged particle. For large enough $p_{\mathrm{T}}$ the toward and away regions predominantly contain particles from the hard $2 \rightarrow 2$ scattering and the transverse region predominantly contains particles from the UE. Figures 2(a) and 2(b) show the charged particle number density (for particles with $p_{\mathrm{T}}>500 \mathrm{MeV}$ and $|\eta|<2.5$ ) in the transverse region as a function of the $p_{\mathrm{T}}$ of the leading charged particle in the event at $\sqrt{s}=0.9$ and $7 \mathrm{TeV}$ respectively. The data are fully corrected for detector effects. The charged particle density increases with $p_{\mathrm{T}}^{\text {lead }}$ from $1 \mathrm{GeV}$ to $\sim 4(6) \mathrm{GeV}$ for $\sqrt{s}=0.9(7) \mathrm{TeV}$. This behaviour is due to the fact that head-on $p p$ collisions will contain more parton-parton interactions and are hence more likely to have one hard collision. The distribution reaches a plateau at this point, as once collisions 


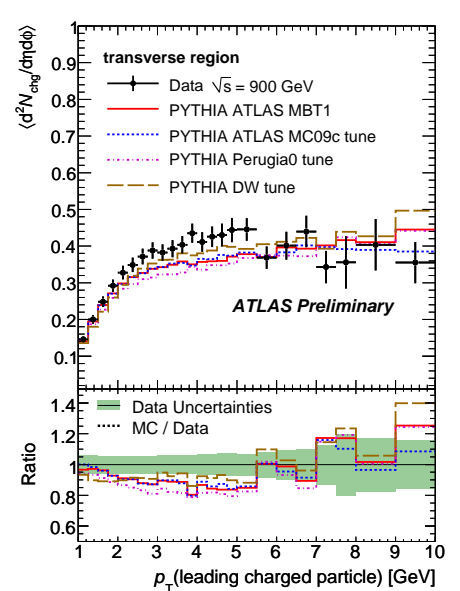

(a)

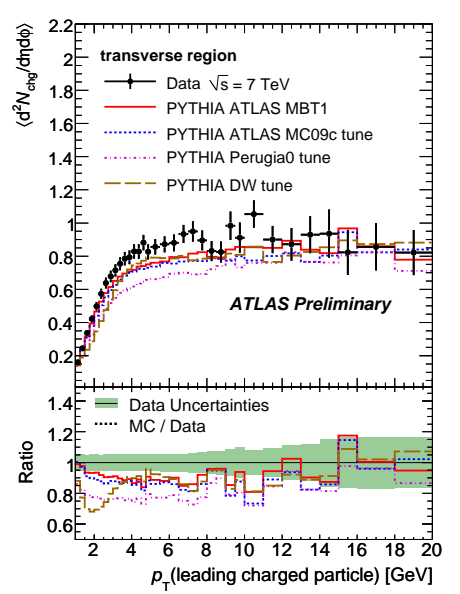

(b)

Figure 2: Charged particle densities in the transverse region as a function of the $p_{\mathrm{T}}$ of the leading charged particle in the events at $\sqrt{s}=0.9$ (a) and 7 (b) TeV.

are head-on the amount of UE activity does not increase with $p_{\mathrm{T}}^{\text {lead }}$.

The PYTHIA6 tunes reproduce this general behaviour, but they all predict less UE activity than the data at both $\sqrt{s}=0.9$ and $7 \mathrm{TeV}$. The statistics of the data in the plateau are not sufficient to determine if the MC tunes are too low in this region. Since this was the region used in the AMBT1 tune, the improvement of the description of the data is minimal. A full description of this data analysis can be found in Ref. [4].

\subsection{Diffraction enhanced minimum bias}

The fraction of diffractive events in the MB data is constrained by studying the ratio $R_{s s}=$ $\frac{N_{s s}}{N_{s s}+N_{d s}}$, where $N_{s s}$ is the number of events with activity on only one MBTS side and $N_{d s}$ is the number of events with activity on both MBTS sides. In order to reduce non-collision backgrounds, one track with $p_{\mathrm{T}}>500 \mathrm{MeV}$ and $|\eta|<2.5$ is required in both cases. Figure 3(a) shows the $R_{s s}$ value measured in the data compared to the predictions from PYTHIA6, PYTHIA8 [5] and PHOJET [6] shown as a function of the ratio of diffractive (SD+DD) cross sections to the total inelastic cross section, where a clear correlation is seen. In all three cases a ratio of cross sections of about 0.3 is preferred, which is in good agreement with the default in both PYTHIA6 and PYTHIA8 (the default values are indicated by the dots on the curves).

The track multiplicity and $p_{\mathrm{T}}$ distributions are shown in Figures 3(b) and 3(c) respectively for the diffraction enhanced sample (those events with activity in only one of the MBTS disks). Note that these distributions have not been corrected for detector effects and are therefore compared to MC samples passed through a detector simulation. PYTHIA8 and PHOJET both do a good job of reproducing the track multiplicity distribution, whereas PYTHIA6 predicts less tracks than observed. This is expected as PYTHIA6 does not include a model of hard diffractive interactions. PHOJET also does an excellent job describing the $p_{\mathrm{T}}$ distribution, whereas PYTHIA8 predicts a too soft distribution. As expected, PYTHIA6 undershoots the data, except in the region $p_{\mathrm{T}}>$ 


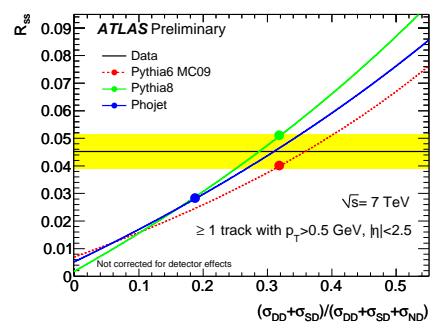

(a)

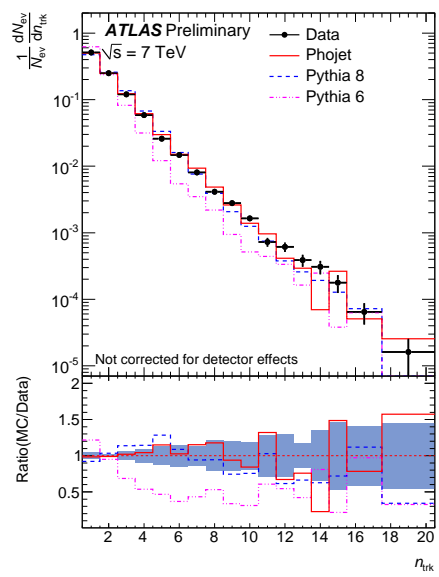

(b)

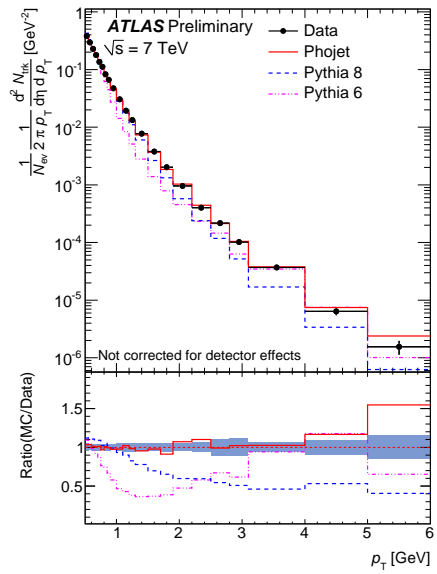

(c)

Figure 3: (a) $R_{S S}$ distribution for data and MC predictions as a function of the relative diffractive crosssection. Track multiplicity (b) and $p_{\mathrm{T}}$ (c) distributions in a diffraction enhanced event sample.

$3 \mathrm{GeV}$, where the ND process contributes a significant amount. A full description of this data analysis can be found in Ref. [7].

\section{Conclusions}

Charged particle distributions from $p p$ collisions and UE distributions at $\sqrt{s}=0.9$ and 7 $\mathrm{TeV}$ measured in the ATLAS detector have been presented. MB distributions are shown in both diffraction suppressed and diffraction enhanced event samples. A new PYTHIA6 ATLAS tune to the diffraction suppressed data sample is presented which significantly improves the description of the high $n_{\mathrm{ch}}$ and high $p_{\mathrm{T}}$ tails as well as the $\left\langle p_{\mathrm{T}}\right\rangle \mathrm{v} s . n_{\mathrm{ch}}$. The PYTHIA6 tunes all under-predict the amount of UE activity at both $\sqrt{s}=0.9$ and $7 \mathrm{TeV}$. Studies of the diffraction enhanced data sample favour the relative diffractive cross section used by PYTHIA, whereas the charged particle distributions are best described by PHOJET.

\section{References}

[1] T. Sjostrand, S. Mrenna and P. Z. Skands, JHEP 0605 (2006) 026.

[2] ATLAS Collaboration, G. Aad et al., JINST 3 (2008) S08003994.

[3] ATLAS collaboration, ATLAS-CONF-2010-031.

[4] ATLAS collaboration, ATLAS-CONF-2010-029.

[5] T. Sjostrand, S. Mrenna and P. Z. Skands, Comput. Phys. Commun. 178 (2008) 852.

[6] R. Engel, Z. Phys. C66 (1995), 203-214.

[7] ATLAS collaboration, ATLAS-CONF-2010-048. 